\title{
A Retrofitted Metallurgical Microscope Using Light Emitting Diodes for Multi-Spectral Imaging
}

\author{
Jerry Opoku-Ansah ${ }^{1}$, Benjamin Anderson ${ }^{1}$, Moses J. Eghan ${ }^{1}$, Peter Osei-Wusu Adueming ${ }^{1}$, Charles L.Y. \\ Amuah $^{1} \&$ Samuel S. Sackey ${ }^{1}$
}

${ }^{1}$ Laser and Fibre Optics Centre (LAFOC), Department of Physics, School of Physical Sciences, College of Agriculture and Natural Sciences, University of Cape Coast, Cape Coast, Ghana

Correspondence: Jerry Opoku-Ansah, Laser and Fibre Optics Centre (LAFOC), Department of Physics, School of Physical Sciences, College of Agriculture and Natural Sciences, University of Cape Coast, Cape Coast, Ghana. Tel: 233-332-133-773. E-mail: jopoku-ansah@ucc.edu.gh

Received: May18, 2017

Accepted: June 12, 2017

Online Published: August 7, 2017

doi:10.5539/mas.v11n9p30

URL: https://doi.org/10.5539/mas.v11n9p30

\begin{abstract}
Multi-spectral imaging (MSI) has made diagnosis of microscopic samples considerably easier and information abound. Most MSI systems use continuum light sources and filters for imaging purposes. However, these light sources and filters are relatively expensive, unstable due to extreme pressure and temperature and associated with prolong acquisition time. In this work, we present a metallurgical microscope retrofitted with light-emitting diodes (LEDs) as illumination sources for MSI microscopy. This multispectral LED imaging microscope (MSLEDIM) is relatively cheaper and capable of acquiring images in reflection, transmission and scattering modes at thirteen (13) different wavelengths ranging from ultraviolet to near infrared. The microscope has been demonstrated in biomedical and entomological research fields. The MSLEDIM can be used in various scientific research fields for imaging microscopic samples.
\end{abstract}

Keywords: multi-spectral imaging, metallurgical microscope, light emitting diodes, microscopy

\section{Introduction}

Multi-spectral imaging (MSI) has been suggested as a non-destructive method used in many applications to quantify biological processes (Hu et al., 2005; Frey \& Warda, 2008; Teikari, 2008; Bautista \& Yagi, 2011; Cosentino, 2015). MSI is the technique of imaging samples using more than one (1) wavelength, extracting spatial as well as spectral data information out of the samples (Hu et al., 2005; Park et al., 2007; Cosentino, 2015; Shrestha \& Hardeberg, 2015). Some of its applications include magnetic resonance imaging (MRI), biometric pattern recognition, retinal physiology, non-image forming (NIF) responses of light, plant physiology, accurate colour reproduction, colour enhancement, digital staining and soil texture determination. On the microscopic level, MSI reveals symptoms of hidden diseases in specimens such as cells and tissues (Prasad, 2003; Lichtman \& Conchello, 2005; Coffey, 2012; Merdasa et al., 2013; Opoku-Ansah et al., 2014).

For years, most MSI systems use broad light sources and filters for microscopy. This is done by selectively transmitting and imaging light of different wavelengths from the broad light source of the microscope. During MSI application in microscopy, the selective transmission of light with different wavelengths by the filters depends on the stability of the spectrum of light source used and the time required during acquisition. However, the spectra produced by most light sources used in MSI microscopy are unstable due to extreme pressure and temperature. The filters used may also degrade with time. Besides, there is very little information about the chemical nature of the investigated samples when using the filters due to lack of spectral content in the images. More so, the light sources have limited number of spectral bands with relatively long acquisition time. This introduces uncertainties which lead to sample photo-bleaching and photo-kinetics, which may cause non-linear absorption and fluorescence (Brydegaard et al., 2011).

Light-emitting diodes (LEDs) as illumination light sources in MSI microscopy have made imaging easier and cost effective (Schubert, 2006; Brydegaard et al., 2011). Also, the stability of spectrum has been improved; modulation speed has increased to the sub-nanosecond scale and with a major reduction in losses during illumination (Herman et al., 2001; Cole \& Turner, 2008; PicoQuant, 2011). LED Wavelengths currently available range from $240 \mathrm{~nm}$ to $7 \mu \mathrm{m}$ (Roithner Laser Techniques, 2011). 
In this work, we present a retrofitted metallurgical microscope which uses nine (9) LEDs emitting a total of 13 wavelengths ranging from $375 \mathrm{~nm}$ to $940 \mathrm{~nm}$. These are used as illumination light sources for MSI in reflection, transmission and scattering modes.

\section{Instrumentation}

Components used for retrofitting the metallurgical microscope are classified into three main categories, namely: mechanical, optical and optoelectronic components.

\subsection{Mechanical Components}

The mechanical components of the MSLEDIM are the main metallic frame of the metallurgical microscope (SP-80, Brunel Microscopes Limited, 2009), three plastic holders for holding LED chips and a brass tube. The plastic holders have a heat sink, locking ring, and contact carrier assembly. The brass tube is made from brass chemically treated to remove its reflective surface.

\subsection{Optical Components}

A fibre ring (FB), consisting of a number of fibres bundled together and guided from a glass-polished end-face into anodized, aluminum housing, is used to diffuse the light in $360^{\circ}$ around the central axis. An optical objective (Reflx ${ }^{\mathrm{TM}}$, Edmund Optics Inc.) based on reflective optics, with amagnification factor $\mathrm{x} 15$ and numerical aperture (NA) 0.28 , is used for collecting and focusing of the light. This objective produces superior quality images over a broad spectral range. An optical diffuser (Edmund OpticsInc.) is used to achieve Lambertian distribution of light. Three (3) sets of 9 LEDs each were used as illumination light sources in reflection, transmission and scattering modes respectively. One of the LEDs emit 3 central wavelengths; $470 \mathrm{~nm}, 525 \mathrm{~nm}$ and $810 \mathrm{~nm}$, while 2 others emit 2 central wavelengths each; $375 \mathrm{~nm}$ and $625 \mathrm{~nm}$, and $400 \mathrm{~nm}$ and $850 \mathrm{~nm}$ respectively. Six LEDs emit single wavelengths at $435 \mathrm{~nm}, 590 \mathrm{~nm}, 660 \mathrm{~nm}, 700 \mathrm{~nm}, 750 \mathrm{~nm}$ and $940 \mathrm{~nm}$.

\subsection{Optoelectronic Components}

A complementary metal-oxide-semiconductor (CMOS) imager (Guppy F-503B, NT63-371, Allied Vision Technologies ${ }^{\odot}$ ), with a resolution of $2592 \times 1944$ pixels was used for image acquisition. The power of the CMOS imager is supplied via IEEE-1394 cable by a laptop computer (PC). National instrument data acquisition device (NI-DAQ), (model USB-6009, National Instruments ${ }^{\mathcal{O}}, 2009$ ) was used to bridge the computer and switching of the LEDs. The DAQ has the ability to display both analogue and digital signals between $0-5 \mathrm{~V}$ in several channels and also the ability to retrieve an analogue signal.

\subsection{Assembling}

Figure 1 shows a schematic diagram of a retrofitted MSLEDIM. The LEDs were arranged and soldered unto 3 stripboards (LED mounts) to serve as the illumination light sources for reflection (M1), transmission (M2) and scattering (M3) modes. Three optical diffusers D1, D2 and D3 were placed at a common point of the optical axes of the LEDs in the plastic holders.

The LED mounts were then placed into the three holders. The holder for M1 mode was placed at the original light position, the second was fixed at the bottom to serve as illumination light source for M2 mode while the third was placed at the back, below the microscope handle, as light source for M3 mode. Resistors, transistors, operational amplifiers and diodes were put together on the stripboard to form an electronic circuit board (ECB) which controls the LEDs. This was placed beneath the microscope and linked to the various plastic holders with connectors. The NI-DAQ was also placed beneath the microscope with the input and output channels connected to the ECB. A binocular head which contains the eyepiece lenses was removed and a piece of black tape used in its place to prevent stray light. One end of the FB was placed at the emanating point of the transmission light source while the other end was linked to the holder for M3 mode. The trinocular head of the microscope was removed and replaced with the camera which was mounted on a support. The signals from the imager are controlled via the laptop computer using a developed Matlab program (R2014a Matlab 8.3.0.532, Mathworks Inc.). The user interface of this program is shown in Figure 2. 


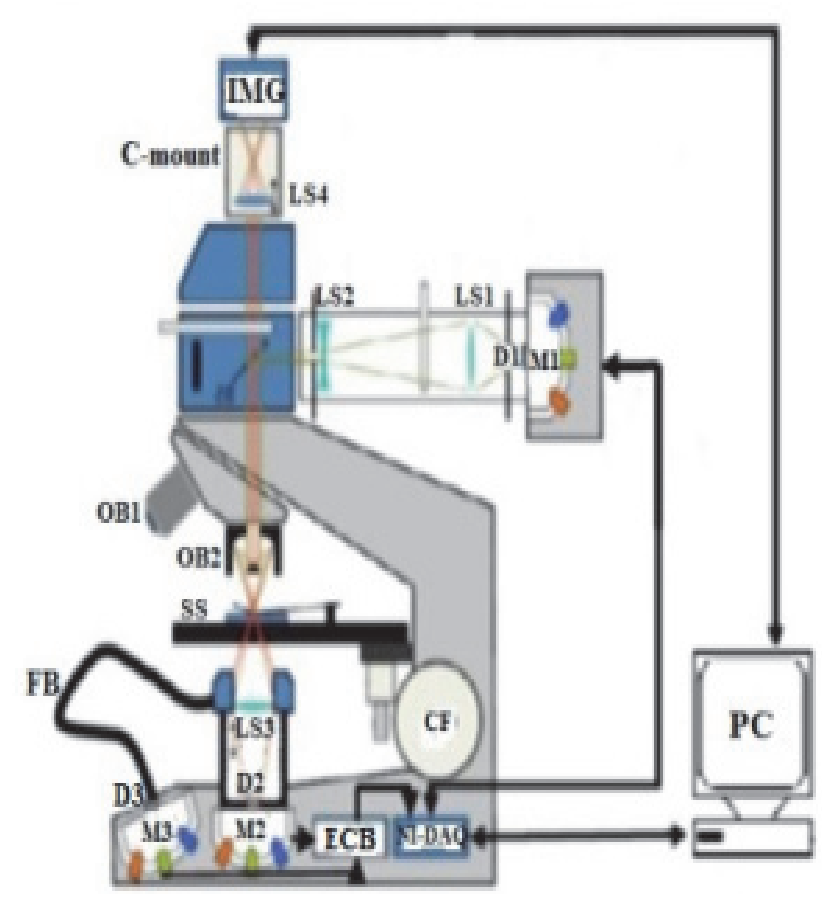

Figure 1. Schematic diagram of the retrofitted metallurgical microscope using light emitting diodes for multi-spectral imaging. Three optical geometries M1, M2 and M3 provide multispectral illumination from the UV to NIR regions in reflection, transmission, and scattering modes respectively. The light from the sample mounted on the sample stage, SS, is collected through a reflective objective OB2. Detection is generally performed by a monochromatic CMOS imager, IMG

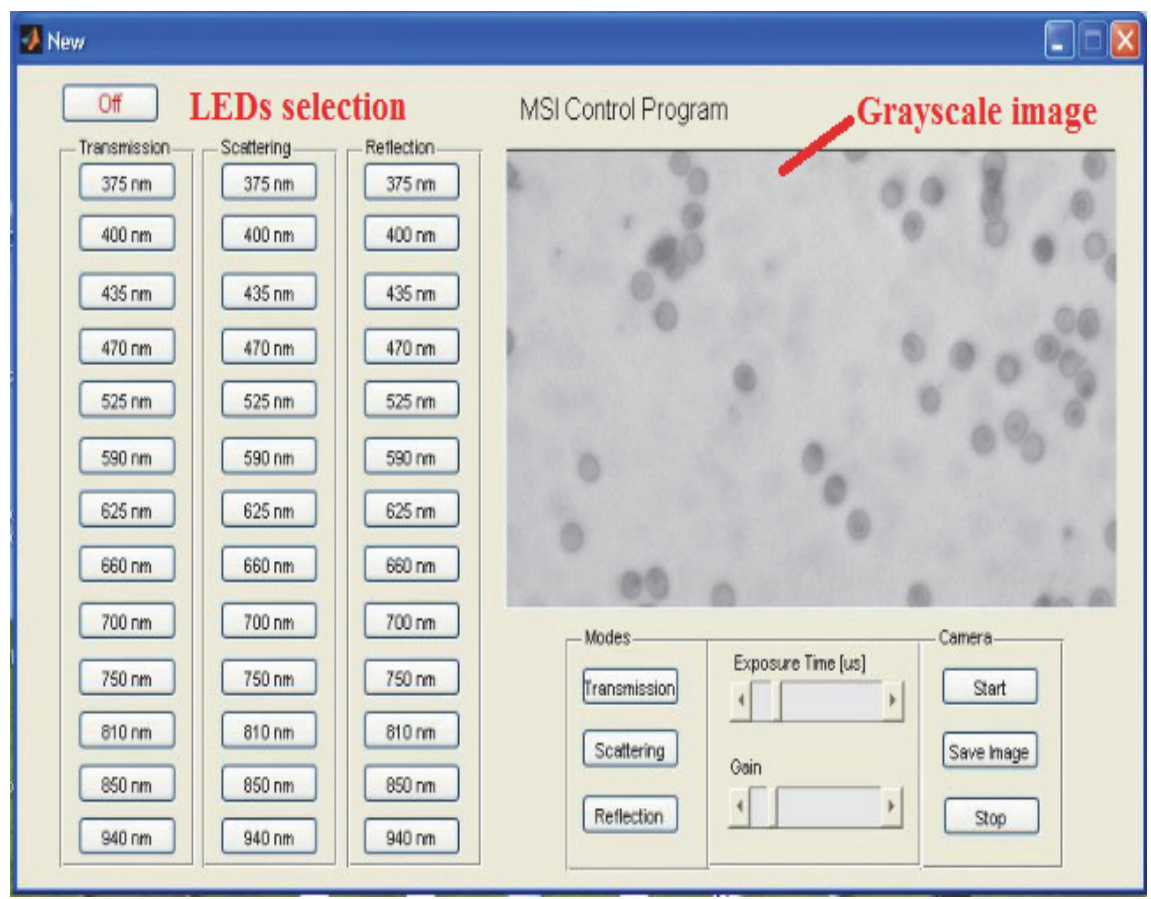

Figure 2. Graphical User Interface (GUI) of Matlab program for controlling signals and capturing images from the imager 


\subsection{Mode of Operation}

A Universal serial bus (USB) from the imager and the NI-DAQ are plugged into USB ports on the computer. The power supply and the computer are then switched on, and the Matlab program launched from the computer. M1, M2 and M3, representing reflection, transmission and scattering modes respectively, are then selected from the geometry icon. Light from the LEDs in M1 mode is scattered by a diffuser (D1), and brought onto focus by a convex lens (LS1) and then diverged by a concave lens (LS2). The light then incidents on the beam splitter (BS) from which $50 \%$ is reflected towards the sample on the sample stage (SS) and the rest transmitted. The reflected light then moves through the Refl $\mathrm{x}^{\mathrm{TM}}$ objective (OB2), reflects on the sample and directed through the BS again. At the same time, $50 \%$ of the reflected light is reflected towards M1 while the other transmitted $50 \%$ is focused by a convex lens (LS4) towards the imager and captured by it via the computer (a 32-bit Intel (R) Core (TM) 2 Duo CPU Toshiba Satellite Pro laptop with processing speed of $2.00 \mathrm{GHz}$ ). Similarly, light from the LEDs in M2 mode is scattered by a diffuser (D2) and focused by a convex lens (LS3) onto the sample where the light is transmitted through the sample on SS. The transmitted light is focused by LS4 towards the imager to be detected and captured by the imager using the computer. In the M3 mode, light from the LEDs is scattered by a diffuser (D3), passed through the FB and scatterd around SS before illuminating the sample on SS. The transmitted light via the sample then passes through OB2 which brings it into focus before being incidenced on BS, which divides the light into two equal halves. The transmitted light is brought to a focus by LS4 after which it is detected by the imager and captured using the computer.

\section{System Testing}

Figure 3 shows spectral sensitivity range of the Guppy F-503B CMOS imager. The figure shows the 13 wavelengths range of the LEDs in the MSLEDIM. The highest spectral sensitivity peak is located at $590 \mathrm{~nm}$. This renders the imager more sensitive to light at this wavelength, resulting in images of samples captured (at this wavelength) to be brighter than those captured at the other wavelengths.

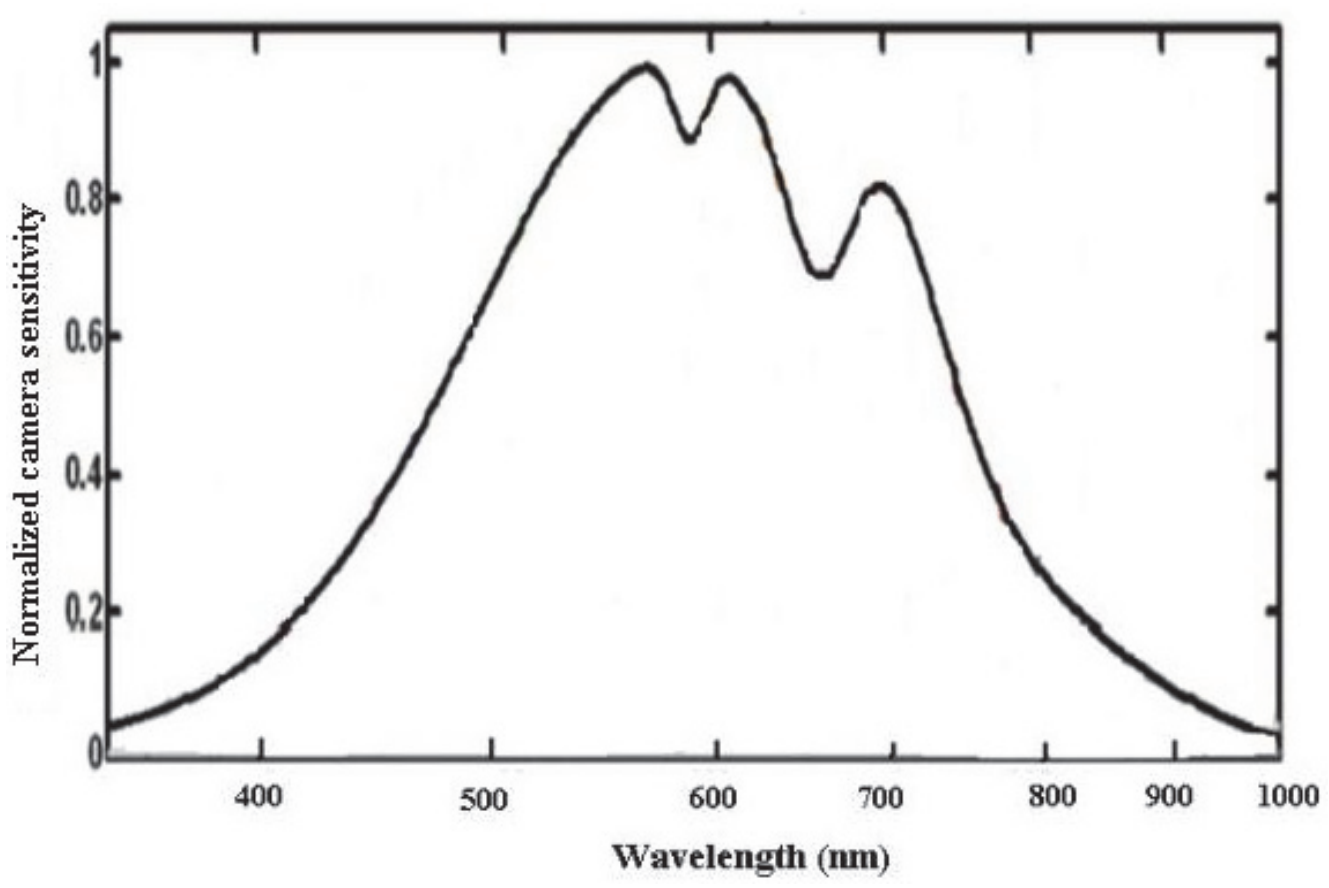

Figure 3. Spectral sensitivity range of the Guppy F-503B CMOS camera

As a way of testing the MSLEDIM, the images of film slides containing blood-smear Plasmodium falciparum parasites infected red blood cells $(i \mathrm{RBCs})$, wing of a bee, white kidney, maize pollen and human skin were acquired. Figures 4 and 5 show grayscale images of $i$ RBCs and wing of a bee on film slides using $590 \mathrm{~nm}$ illumination LED source acquired in transmission, scattering and reflection modes respectively. 

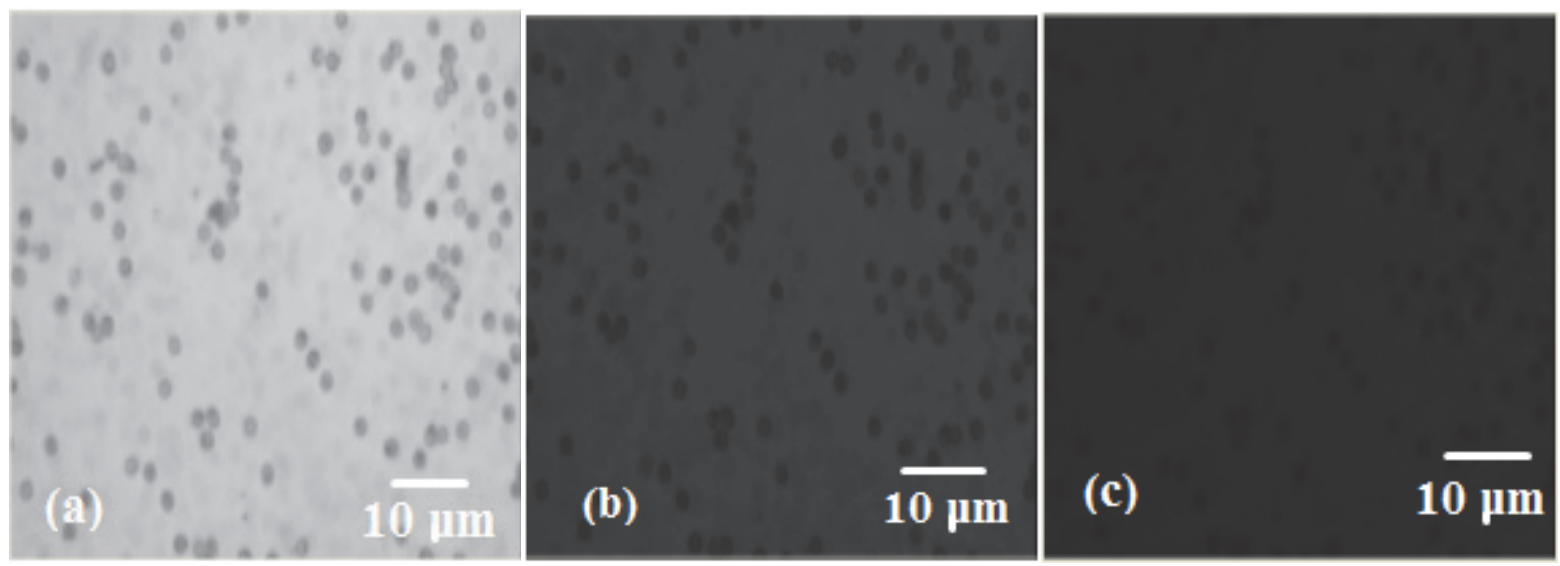

Figure 4. Grayscale images acquired using the retrofitted metallurgical multispectral light-emitting diodes imaging microscope with $590 \mathrm{~nm}$ illumination source in (a) transmission, (b) scattering and (c) reflection modes.

The dark spots in the images represent Plasmodium falciparum infected RBCs
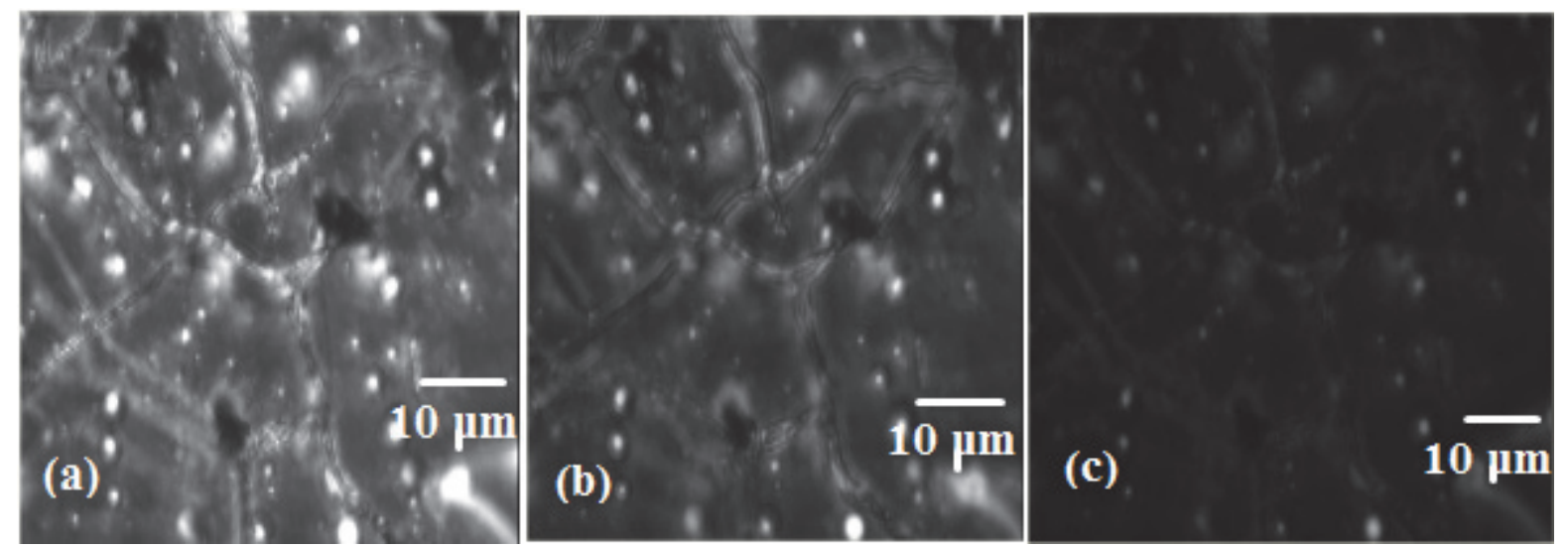

Figure 5. Grayscale images acquired using the retrofitted metallurgical multispectral light-emitting diodes imaging microscope with $590 \mathrm{~nm}$ illumination source in (a) transmission, (b) scattering and (c) reflection modes. The thin lines in the images represent veins in the wing of a bee

Figures 4 and 5 show non-uniform background images interspersed with dark spots representing the $i$ RBCs and bright spots depicting veins in the wing of a bee respectively. These images have the same dimensions but different grayscales. The other images (not shown) have the same dimensions as in Figures 4 and 5 , and also show different grayscales. The different grayscales are as a result of the selective spectral bands of absorption of the LED sources. Figures 6-10 show pixel histogram of the Red (R), Green (G) and Blue (RGB) colours of $i$ RBCs, wing of a bee, white kidney, maize pollen and human skin respectively. This gives a graphical illustration of the light distribution of the digital images. 3-D scatter plots of the RGB colours are presented in Figure 11. There are significant differences in the RGB-pixel histogram and the scatter plot of all the samples. Such quantitative information lends itself to discrimination algorithms using RGB. The $i$ RBCs and human skin images (Figures 6 and 10) show a very unique RGB pixel histogram depicting high pixel intensities for R and B colours. This could be attributed to the high reflectivity of $R$ and $B$ colours which dominates over $G$ colour in these samples. The wing of a bee image (Figure 7) shows a pixel histogram with high pixel intensities for B colour only. This means that the B colour is significant in the veins of the wing of the bee. The $i$ RBCs, human skin and wing of a bee images show broader pixel histogram for the B colour. This could also be attributed to the reflectivity of the $B$ colour to the human eye which dominates over the $R$ and $G$ colours in these samples. The RGB scatter plots of $i$ RBCs, wing of a bee, white kidney and human skin show slightly high scattering of the pixel intensities than that of maize pollen. This observation could be attributed to the uneven light intensities transmitted by the imager to the $i \mathrm{RBCs}$, wing of a bee, white kidney and human skin on the sample stage. Besides, the scatter plots show majority of the pixel intensities of $i$ RBCs, wing of a bee, white kidney and human skin in the $\mathrm{G}$ and $\mathrm{B}$ colour regions indicating the sensitivity of these samples in the RGB colour domain. 

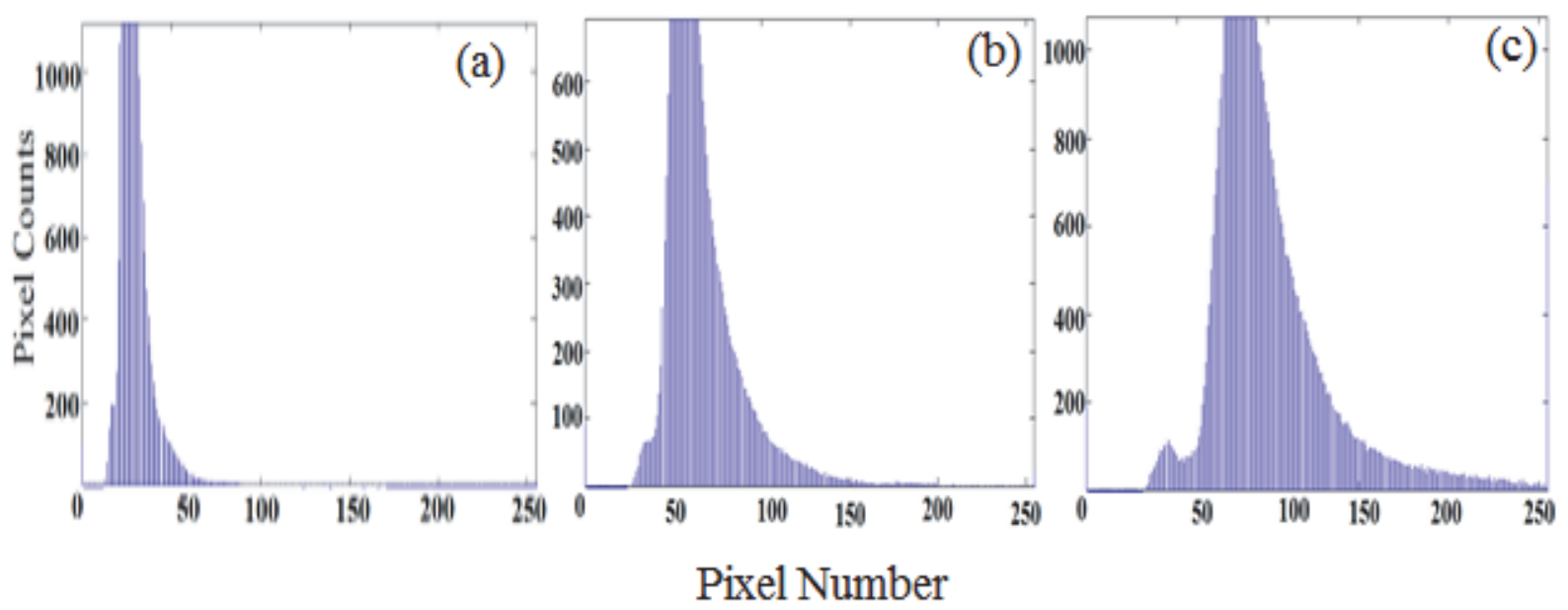

Figure 6. (a) Red (R), (b) Green (G) and Blue (B) RGB-pixel histogram of intensities extracted from an image of a blood smear slide containing Plasmodium falciparum parasites in infected RBCs ( $i \mathrm{RBCs})$. The image was acquired using the retrofitted metallurgical microscope
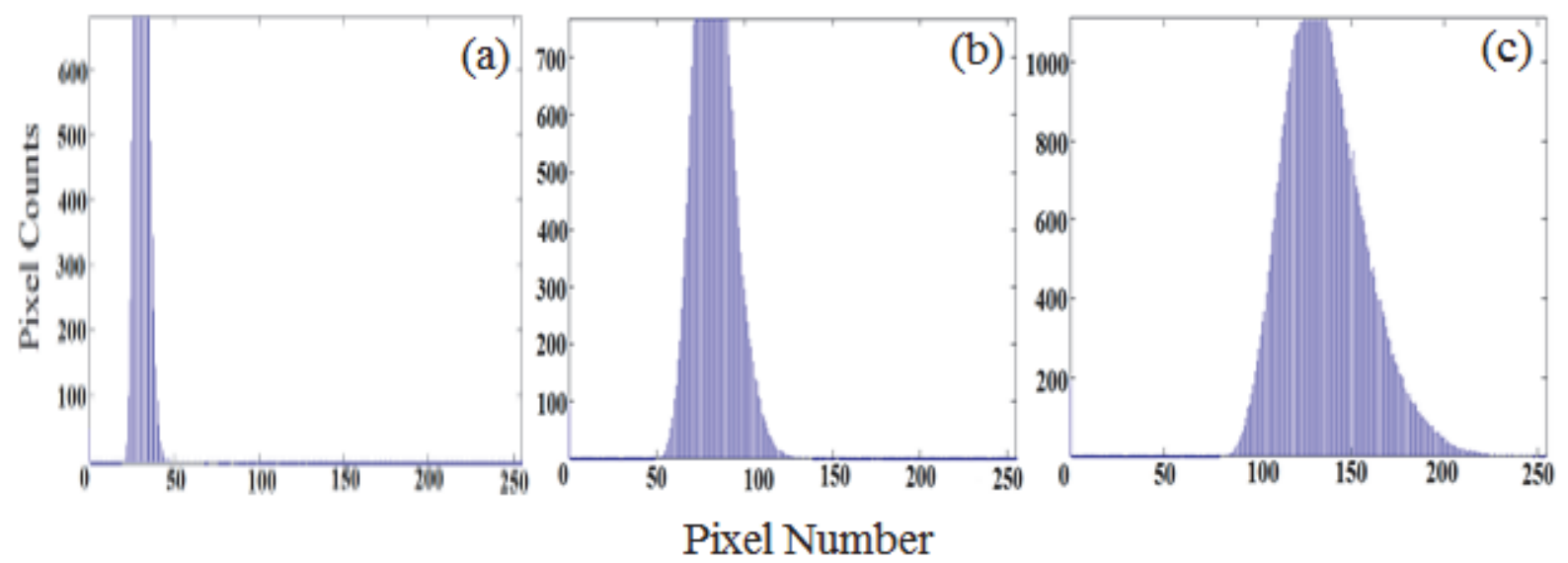

Figure 7. (a) Red (R), (b) Green (G) and Blue (B) RGB-pixel histogram of intensities extracted from an image of a slide containing wing of a bee. The image was acquired using the retrofitted metallurgical microscope
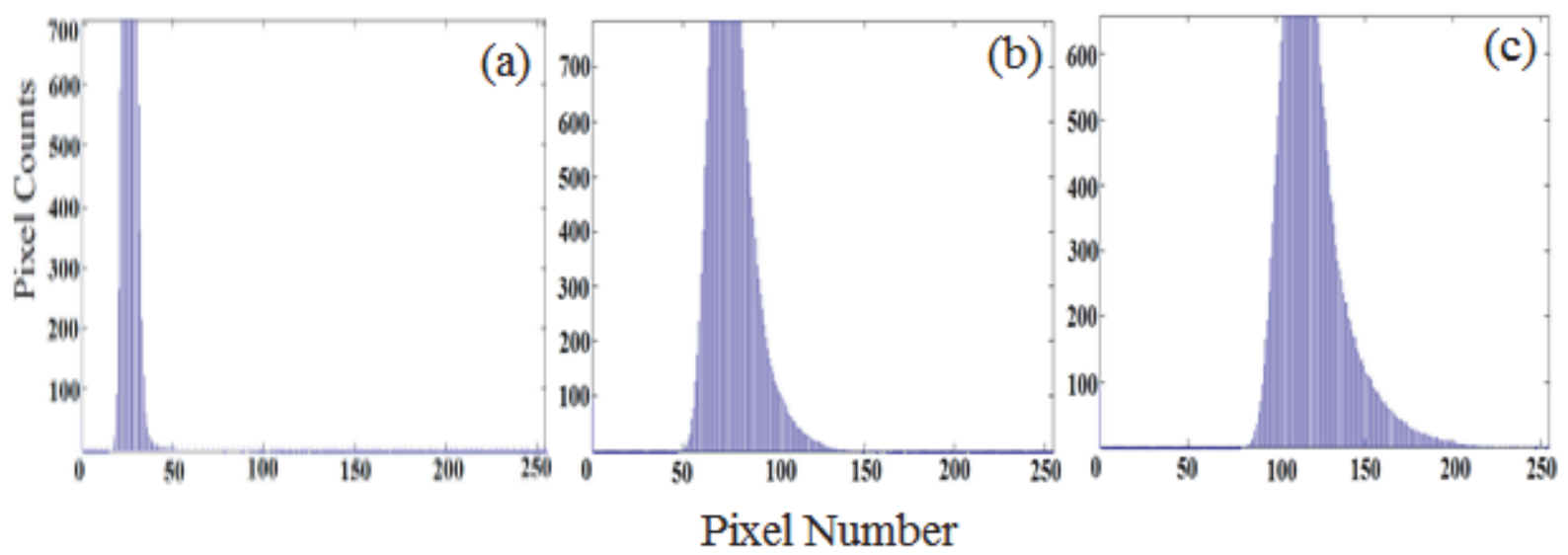

Figure 8. (a) Red (R), (b) Green (G) and Blue (B) RGB-pixel histogram of intensities extracted from an image of a slide containing a white kidney. The image was acquired using the retrofitted metallurgical microscope 

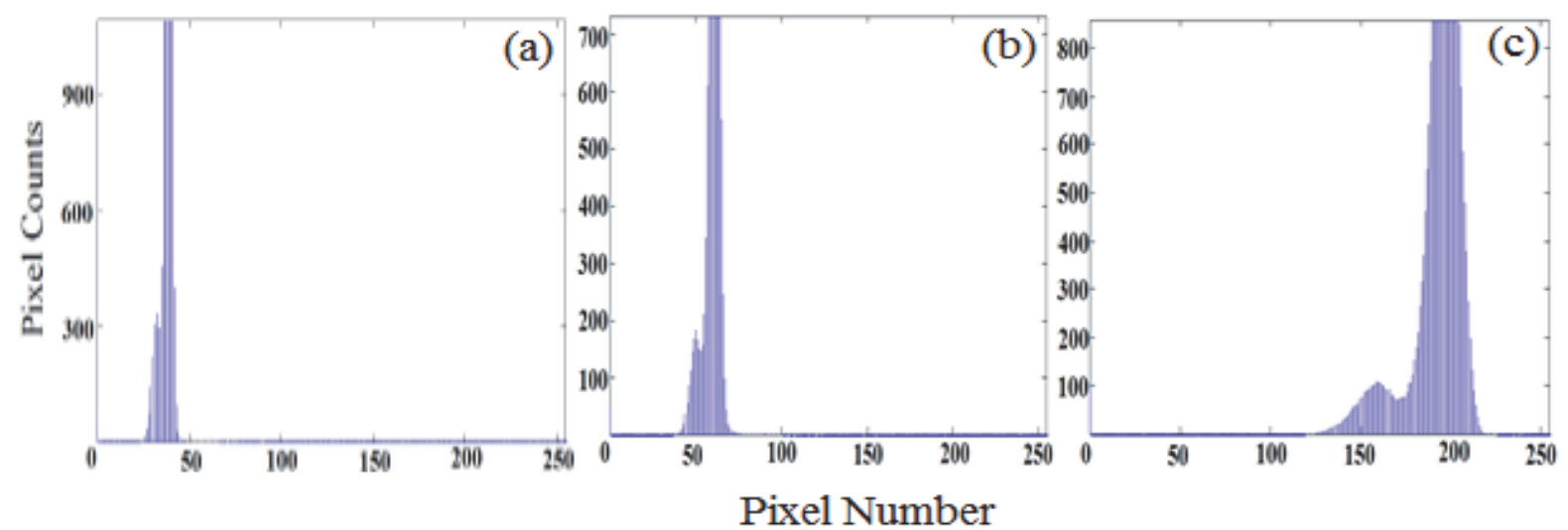

Figure 9. (a) Red (R), (b) Green (G) and Blue (B) RGB-pixel histogram of intensities extracted from an image of a slide containing a maize pollen. The image was acquired using the retrofitted metallurgical microscope
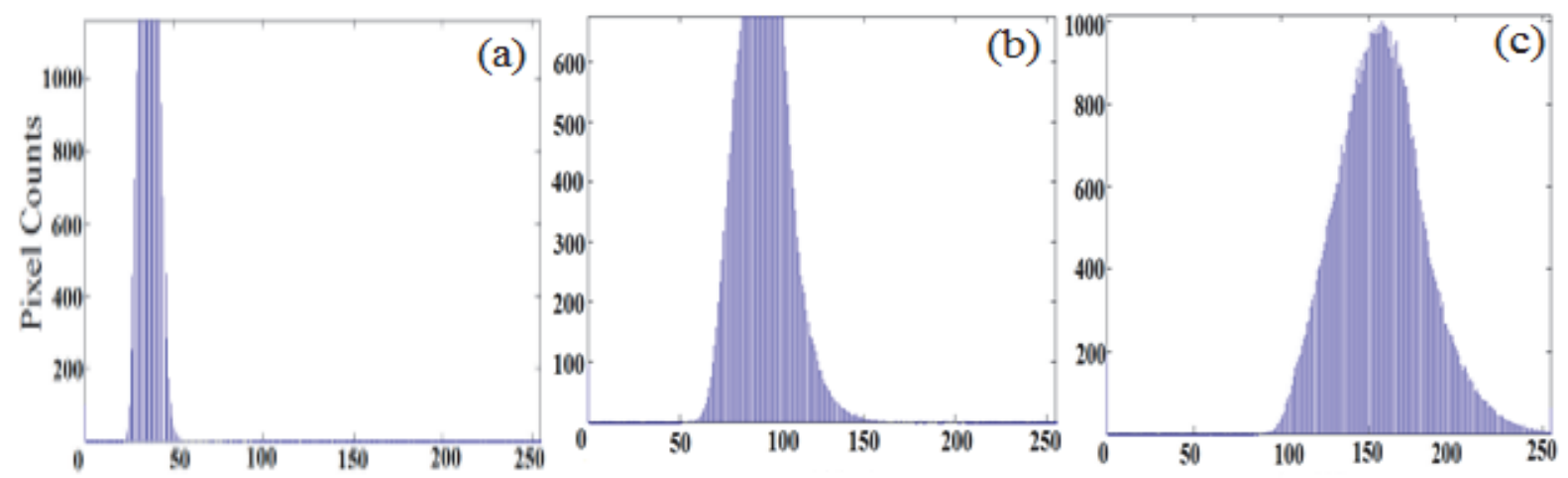

Pixel Number

Figure 10. (a) Red (R), (b) Green (G) and Blue (B) RGB-pixel histogram of intensities extracted from an image of a slide containing a human skin. The image was acquired using the retrofitted metallurgical microscope
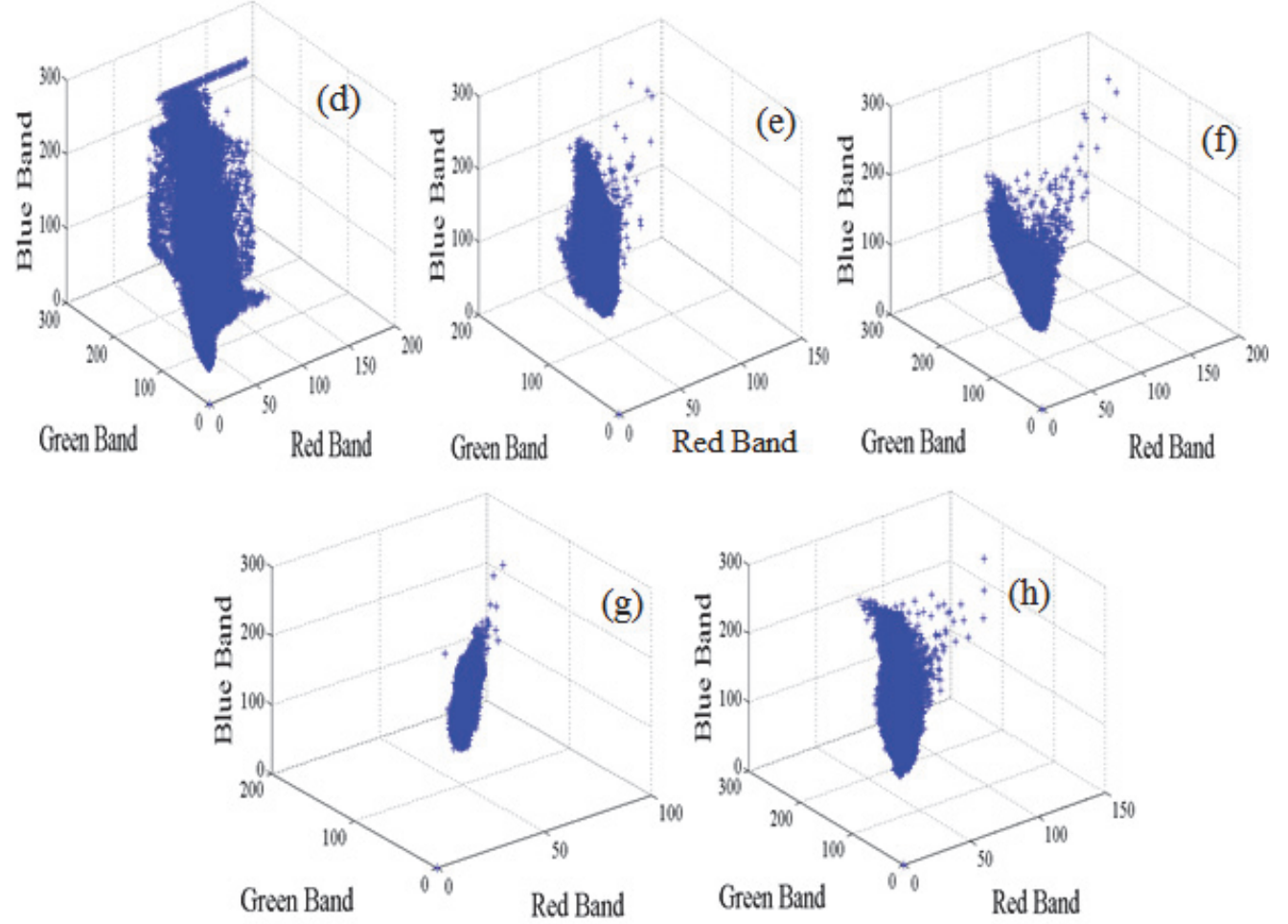

Figure 11. Scatter plot showing RGB pixel intensities of images representing (d) $i \mathrm{RBCs}$, (e) wing of a bee, (f) white kidney, (g) maize pollen and (h) human skin 


\section{Conclusions}

Light sources used in MSI microscopy have restricted number of spectral bands associated with comparatively long acquisition times. This leads to sample photo-bleaching and photo-kinetics and ultimately unstable spectra. A retrofitted metallurgical microscope has been demonstrated for multi-spectral imaging. This new microscopic imaging system uses a relatively inexpensive set of 9 LEDs each as illuminating sources in reflection, transmission and scattering modes respectively. Each set of LEDs emits 13 wavelengths as light sources and is capable of acquiring megapixels microscopic images ranging from UV to NIR, making the imaging system an attractive tool for research. Images from this microscope are in grayscale colour model, which is device-independent and therefore colour information is unaffected by intensity manipulation. The results from the demonstrations show that the system is versatile and can potentially be used in biological and biomedical research.

\section{Acknowledgements}

The authors wish to express their appreciation to the International Programme for Physical Sciences (IPPS), International Sciences Programme (ISP), Uppsala University, Sweden and Office of External Activities (OEA) of ICTP, Trieste, Italy for funding and donation of microscope. The efforts of all members of the African Spectral Imaging Network (AFSIN) and the Laser Fibre Optics Centre (LAFOC) research group members are appreciated.

\section{References}

Bautista, P. A., \& Yagi, Y. (2011). Localization of Eosinophilic Esophagitis from H\&E stained images using multispectral imaging. Diagnostic Pathology, 6, 1-8. http://dx.doi.org/10.1186/1746-1596-6-S1-S2

$\begin{array}{lllll}\text { Brunel Microscopes } & \text { Ltd. } & \text { (2009). } & \text { Retrieved }\end{array}$ http://www.brunelmicroscopes.co.uk/metallurgical-microscopes.html

Brydegaard, M., Merdasa, A., Jayaweera, H., Ålebring, J., \& Svanberg, S. (2011). Versatile multispectral microscope based on light emitting diodes. Review of Scientific Instruments, 82, 1106-1113. https://doi.org/10.1063/1.3660810

Coffey, V.C. (2012). Multispectral imaging moves into the mainstream. Optics and Photonics News, 23, 18-24.https://doi.org/10.1364/OPN.23.4.000018

Cole, R. W., \& Turner, J. N. (2008). LEDs as illumination and detection sources inmicroscopy. Microanalysis, 14, 243-250. https://doi.org/10.1017/S1431927608080288

Cosentino, A. (2015). Panoramic, Macro and Micro Multispectral Imaging: An Affordable System for MappingPigments on Artworks. Journal of Conservation and Museum Studies, 13(1), 6, 1-17.http://dx.doi.org/10.5334/jcms.1021224

Herman, P., Maliwal, B.P., Lin, H.J., \& Lakowicz, R. (2001). Exciting bright field fluorescence with power LEDs. Journal of Microscopy, 203,176-181. https://doi.org/10.1046/j.1365-2818.2001.00943.x

Hu, Y., Wu, Q., Liu, S., Wei, L., Chen, X., Yan, Z., Yu, J., Zeng, L. \& Ding, Y. (2005). Study of Rice Pollen Grains by Multispectral Imaging Microscopy, Microscopy Research and Technique, Wiley-Liss, Inc., 68, 335-346. https://doi.org/10.1002/jemt.20256

Lichtman, J. W., \& Conchello, J.A. (2005). Fluorescence microscopy. Nature Methods, 2(12), 910-919.http://dx.doi.org/10.1038/nmeth817

Merdasa, A., Brydegaard, M., Svanberg, S., \& Zoueu, J. T. (2013). Staining-free malaria diagnostics by multispectral and multimodality light-emitting-diode microscopy. Journalof Biomedical Optics, 18(3), 0360021-03600210. https://doi.org/10.1117/1.JBO.18.3.036002

Opoku-Ansah, J., Eghan, M. J., Anderson, B., \& Boampong, J. N. (2014). Wavelength Markers for Malaria (Plasmodium Falciparum) Infected and Uninfected Red Blood Cells for Ring and Trophozoite Stages. Applied Physics Research, 6(2), 47-55.https://doi.org/10.5539/apr.v6n2p47

Park, J. I., Lee, M. H., Grossberg, M. D., \& Nayar, S. K. (2007). Multispectral imaging using multiplexed illumination.IEEE 11th International conference on computer vision, 1-8. Retrieved fromhttp://www1.cs.columbia.edu/CAVE/publications/pdfs/Park_ICCV07.pdf

Pico Quant. (2011). Course on fluorescence techniques. Retrieved from http://www.picoquant.com

Prasad, P. N. (2003). Introduction to biophotonics, Hoboken, NJ: Wiley-Interscience. 
https://doi.org/10.1002/0471465380

Schubert, E. F. (2006). Light-Emitting Diodes (2nd Ed.). Cambridge University Press, 432. https://doi.org/10.1017/CBO9780511790546

Shrestha, R., \& Hardeberg, J.Y. (2015). Multispectral imaging: an application to density measurement of photographic paper in the manufacturing process control. Proc. SPIE 9405, Image Processing: Machine Vision Application VIII, 94050G. https://doi.org/10.1117/12.2083280

\section{Copyrights}

Copyright for this article is retained by the author(s), with first publication rights granted to the journal.

This is an open-access article distributed under the terms and conditions of the Creative Commons Attribution license (http://creativecommons.org/licenses/by/4.0/). 